\title{
NONLINEAR FILTERING APPROACHES TO FIELD MAPPING BY SAMPLING USING MOBILE SENSORS
}

\author{
M. Mysorewala, L. Cheded and A. Qureshi \\ Systems Engineering Department, College of Computer Sciences and Engineering, King \\ Fahd University of Petroleum and Minerals (KFUPM), Dhahran, Saudi Arabia
}

\begin{abstract}
This work proposes a novel application of existing powerful nonlinear filters, such as the standard Extended Kalman Filter (EKF), some of its variants and the standard Unscented Kalman Filter (UKF), to the estimation of a continuous spatio-temporal field that is spread over a wide area, and hence represented by a large number of parameters when parameterized. We couple these filters with the powerful scheme of adaptive sampling performed by a single mobile sensor, and investigate their performances with a view to significantly improving the speed and accuracy of the overall field estimation. An extensive simulation work was carried out to show that different variants of the standard EKF and the standard UKF can be used to improve the accuracy of the field estimate. This paper also aims to provide some guideline for the user of these filters in reaching a practical trade-off between the desired field estimation accuracy and the required computational load.
\end{abstract}

\section{KEYWORDS}

Information fusion, Adaptive Sampling, Environment Mapping, Non-linear Filter, Mobile Sensor

\section{INTRODUCTION}

Mobility in a Wireless Sensor Network (WSN) plays a vital role in distributed monitoring applications and requires that sensors be deployed efficiently with an awareness of the information gain, communication constraints, resource allocation and coordination, and energy utilization. It calls for the use of interconnected nodes in a mobile sensor network-like topology covering the wide geographic spread of the field of interest so as to sense variables of interest such as temperature, humidity, oil and gas, vibrations, etc by using a sampling scheme to collect samples from the spatio-temporal field.

Typical adaptive schemes used include uniform sampling whereby samples are collected from points on a uniform grid that spans across the entire field or random sampling where randomlyselected field points are used as the next sampling locations [15]. In either of these 2 schemes, the process of where to sample next is driven by either a fixed decision (uniform sampling) or a random one (random sampling), and neither of which attempts to exploit an "awareness" of the field being sampled. An interesting alternative to these schemes is provided by Adaptive Sampling (AS) where the selection of the next sample is continuously adapted to real-time environmental changes so as to maximize the information gain. As such, an adaptive sampling scheme aims to make the sampling process informationally-rich, thereby offering a very important energy-saving sampling (or data-acquisition) scheme in applications such as WSN [1]. Some advantages that can accrue from AS include an increase in the sensor node lifetime, and in 
the case of a mobile sensor, it can harness the saved energy to be used for robot motion. An energy-efficient adaptive sampling scheme is also practically attractive in that it leads indirectly to fewer computations and less data communication across the network.

In general, a spatio-temporal distribution can be modeled in a deterministic or stochastic manner. Deterministic white-box parametric models are widely used with the assumption of a stochastic measurement error. Christopoulos and Roumeliotis [2] present an approach for estimating the parameters of a diffusion equation that describes the propagation of an instantaneously-released gas. Five parameters of the diffusion equation are estimated which were the mass of the released gas, the eddy diffusivity constant, the $(x y)$ location of the release point, and the time of explosion. This is done by collecting the samples adaptively by generating either a locally- or globallyoptimal trajectory for the robot movement in order to develop a computationally-efficient approach. Cannell and Stilwell [3] present two approaches for the adaptive sampling of underwater processes for the neutral tracer boundary estimation using Autonomous Underwater Vehicles (AUVs). The first approach assumes a parametric model, while the second one uses an information-theoretic approach. The parametric model approximates the neutral tracer with an ellipse, the parameters of which are estimated using a nonlinear regression or an Extended Kalman Filter (EKF). In the non-parametric approach, the center of mass of the neutral tracer is estimated by a single or multiple AUVs. This approach can be used when an accurate model of the system is not known.

Another approach ([4]) assumes a stochastic process model. Stochastic modeling is used because, for a wide-area environment field, the parameter space becomes huge and mathematically intractable in a deterministic setting.

In our previous work $([5,6])$, we developed an approach to represent a complex nonlinear field with a sum-of-Gaussians field, with the initial estimate of its black-box parameters obtained through a Radial Basis Function (RBF) neural network (NN) trained with initial samples that were collected sparsely. Then we used the standard Extended Kalman Filter (EKF) to improve both the estimate of these parameters and the error covariance through an adaptive sampling strategy [5, 6]. Unlike non-adaptive sampling-based estimation techniques, this EKF-based adaptive sampling framework for complex field estimation makes it possible to start with a lowresolution field representation, then guided by its adaptive sampling scheme, thoroughly sample selected high-variance areas only, thereby maximizing the information gain, while using fewer samples than would otherwise be the case. This in turn leads to a decrease both in computation and communication loads as well as in processing time, and to an increase in energy saving in the overall adaptive sampling process, thereby constituting the first contribution of our two main contributions to this paper. EKF is a standard nonlinear filtering technique for state and parameter estimation, which is achieved through linearization of nonlinear models around an operating point in order to locally apply the linear KF. Several extensions of the standard EKF were introduced because of the problems with the linearization process involved in the EKF, which leads to either a suboptimal performance, or sometimes even to a complete divergence of the filter. These extensions of the standard EKF provide a better state estimation but at the expense of a higher complexity. Some extensions of the standard EKF found in the literature are the Iterated-EKF (iEKF) [7, 16-18], higher-order-EKF [7, 16-18], and Unscented Kalman Filter (UKF) ([8, 1618]).

Our results presented in this paper show that a standard EKF with a high initial uncertainty in the parameters, i.e. a poor initial field estimate, leads to a poor convergence of the field parameter estimates and, in some cases, may even lead to their divergence ([9]). Our second contribution is to remedy this situation through the novel application, analysis and performance evaluation (from the viewpoints of accuracy, sensitivity to initial field estimate as well as initial error covariance, 
and computational effort involved) of 4 traditional nonlinear filters all supplemented with an AS scheme, to the spatial field distribution estimation that involve a much larger number of parameters than other traditional application areas (e.g. radar tracking) do, as explained next.

Radar Signal processing and Target tracking in particular have been a fertile ground for both research into, and application of, nonlinear filtering as witnessed by the vast literature in this area ( see for e.g. [10, 11, 12] and the references therein). However, past work on target tracking using various nonlinear filters $([10,11])$ show that for non-maneuvering targets, filters such as EKF, $\mathrm{UKF}$, standard particle filter (PF), etc give essentially a similar performance, but for maneuvering targets, PF's showed a much better performance. However, unlike in the target tracking problem as well as in the two previously-mentioned real-life applications, where the parameter space is of moderate size, our application deals here with an environmental distribution that is represented with a parametric model which involves a large number of parameters and where computational complexity and estimation accuracy become two crucial issues to consider. As such, the large number of parameters involved therefore gives our application of these 4 traditional nonlinear filters its novel aspect and sets it well apart from other known applications.

This paper is structured as follows: in section 2, we discuss the mathematical background and theoretical performance of the different filters used in this paper; section 3 presents the comparative study of these various filters through simulation; section 4 concludes the paper.

\section{MATHEMATICAL BACKGROUND OF NON-LINEAR ALGORITHMS USED}

In our previous work [5], we generated a complex time-varying fire field (using the well-known fire-spread models) which we considered as our true field to be reconstructed. A low-resolution representation of the true field was acquired and used to train the Radial Basis Function (RBF) NN to model it as a sum-of-Gaussians parametric field. The parameter estimate acquired through training was the parameter vector $X$ (shown in eq. 1) of the Gaussians with uncertainty measure $P$. General process and measurement models were presented in [5], where the process model includes both the robot and field dynamics along with their uncertainty errors which are assumed to be Gaussian and, the measurement model which includes the sensor measurement models involving both the robot and field parameters.

In order to focus our study solely on both the parameter estimation accuracy and computational complexity of the above-mentioned nonlinear filters, we deliberately kept the dynamics of the robots out of the analysis in this paper, henceforth referring to robots as mobile sensors and also consider both the field and the measurement processes stationary. However, throughout this paper our true field is represented by a single snapshot of the field to be mapped since we are not considering the dynamics of the field. As such, the parameters of the true field are considered as constant.

As covered in our previous work, a single-robot-based AS algorithm for a 2D spatially-stationary field $g(x, y)$ shown in figure 1 can be described as follows [5]:

1) Low-resolution sampling: The field $g(x, y)$ of size $m \times m$ is divided into uniform square-sized grids $n \times n$ such that $n<m$, and where samples are collected at the centers of each of the $n \times n$ grids. Hence $m / n \times m / n$ samples are collected as a low-resolution representation of the actual field. 
2) Parameterization: Parametric representation of the field is achieved by training an $N$-neuron RBF neural network with the acquired low-resolution data. This results in a representation of the field as a sum of $N$ Gaussians (one per neuron), and a common offset (or bias) parameter $b$, with each neuron having its own parameters characterizing the Gaussian associated with it, such as its peak amplitude $a_{i}$, variance $\sigma_{i}$ and center coordinates $\left(x_{0 i}, y_{0 i}\right)$.

Mathematically, a spatially- stationary field is represented by the parameter vector $X$ defined by:

$$
X=\left[\begin{array}{llllllllll}
b & a_{1} & \sigma_{1} & x_{01} & y_{01} & \ldots & a_{N} & \sigma_{N} & x_{0 N} & y_{0 N}
\end{array}\right]^{T}
$$

Where $X$ is the vector containing the true values of its component parameters which are not known due to: (i) the resolution error between the actual true field and its acquired low-resolution version, and (ii) the RBF training error. Each parameter in the parameter vector $X$ has an initial estimate value $\hat{X}_{0}$ and an initial error covariance $P_{0}$, which are achieved through $\mathrm{NN}$ training of the low-resolution data.
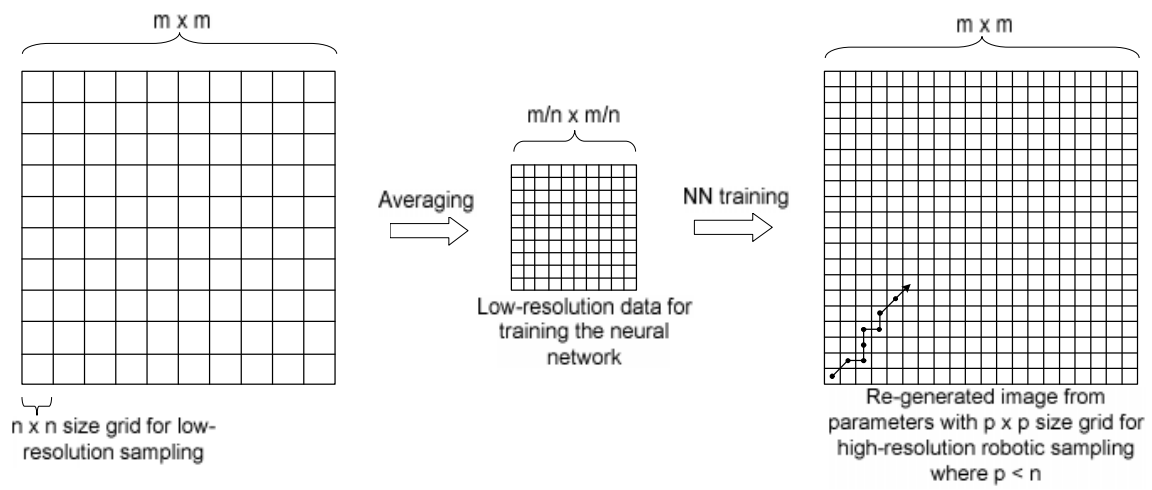

Figure 1. Three steps of Adaptive Sampling algorithm i.e. Low-resolution sampling, Parameterization and High-resolution sampling

3) High-resolution sampling: In order to improve the field estimate, spot measurements are made by a mobile sensor which collects samples $\mathrm{z}_{k}$ in a grid of size $p \times p($ where $p<=n$ ) based on a heuristic Greedy Adaptive Sampling (GAS) algorithm [5]. According to the GAS algorithm, the next sampling location is searched for within the vicinity of the currently-sampled location, based on a criterion of minimization of the norm of the parameter error covariance matrix.

The EKF measurement equation is given by:

$$
z_{k}=g\left(X, x_{k}, y_{k}\right)+v=b+\sum_{i=1}^{N} a_{i} \exp \left[-\sigma_{i}\left\{\left(x_{k}-x_{0 i}\right)^{2}+\left(y_{k}-y_{0 i}\right)^{2}\right\}\right]+v_{k}
$$

Where $v \sim N(0, R)$ is the zero-mean measurement noise with a Gaussian distribution and $R$ as its covariance; the pair $\left(x_{k}, y_{k}\right)$ is the robot location for the $k^{\text {th }}$ sample.

The measurement update for the EKF is given by: 


$$
\begin{aligned}
& P_{k+1}=\left[\left(P_{k+1}^{-}\right)^{-1}+G_{k+1}^{T} R^{-1} G_{k+1}\right]^{-1}, K_{k+1}=P_{k+1} G_{k+1}^{T} R^{-1} \\
& \hat{X}_{k+1}=\hat{X}_{k+1}^{-}+K_{k+1}\left\{z_{k+1}-g\left(\hat{X}_{k+1}^{-}, x_{k+1}, y_{k+1}\right)\right\}
\end{aligned}
$$

Where $\hat{X}_{k+1}$ is the vector containing the parameter estimates at step $(k+1)$ after incorporating the information provided by the measurement $z_{k+1}$. The time-update result of $\hat{X}_{k+1}^{-}$at step $(k+1)$ is equal to $\hat{X}_{k}$, the measurement-update at step $k$, as the parameter vector is assumed to be stationary. $P_{k+1}$ is the parameter error covariance, $G_{k+1}$ is the $g\left(\hat{X}_{k+1}^{-}, x_{k+1}, y_{k+1}\right)$ linearized about the current estimate $\hat{X}_{k+1}^{-}$, and $K_{\mathrm{k}+1}$ is the Kalman gain.

By using the 2-norm of the parameter error covariance as the information measure, the AS algorithm moves the mobile sensor from $\left(x_{\mathrm{k}}, y_{\mathrm{k}}\right)$ to $\left(x_{\mathrm{k}+1}, y_{\mathrm{k}+1}\right)$ such that the 2-norm of the covariance matrix $\left(\left\|P_{k+1}\right\|\right)$ is minimized over the search space.

The vector $X$ in eq (1) shows the parameters of the Gaussians used to model our field, which are the initial offset $b$, peak amplitude of the $i^{\text {th }}$ Gaussian $a_{i}$, its variance $\sigma_{i}$, and its center at $\left(x_{0 i}, y_{0 i}\right)$, for $i=1, \ldots, N$. The total number of parameters used is equal to $(4 N+1)$. The selection criteria for the number of neurons and their effect on the training error have been discussed in our previous work [5]. In summary, using too small a number of neurons $(N)$ would lead to an undertraining of the $\mathrm{NN}$ and would therefore not capture the complete dynamics of the field. It would hence result in a huge initial error that cannot be reduced later even by taking more highresolution samples. On the other hand using too large a value of $N$ would result in an overparameterized model and hence to an over-trained $\mathrm{NN}$, or at least would make the algorithm unnecessarily more complex than is needed. The initial estimates of these parameters are acquired by training the RBF NN using the initial sparsely-collected samples. In our previous work [5, 6, 13], we have considered several different scenarios, with different field complexities including stationary fields such as linear, sum-of-Gaussians and complex stationary fire field, as well as time-varying complex fire field [5]. We have also tested different sampling approaches such as raster (row-by-row) scanning, adaptive sampling, and adaptive sampling with greedy heuristics [6], using single and multiple robots with centralized and distributed schemes [13]. We have also performed an accuracy analysis of initial field estimates when different grid sizes (and hence different number of samples) were considered to train the $\mathrm{NN}$ with a different number of neurons. Once the initial parameter estimates are acquired by $\mathrm{NN}$ training, the parameter estimates are further improved by taking and processing the current sensor measurement, shown in eq. 2 above using the EKF-based AS algorithm.

In this paper, the focus is on investigating the convergence properties on some nonlinear filters (iEKF, SOEKF, and UKF) used as alternatives to the standard EKF and to demonstrate their novel use in important applications such as complex field estimation and mapping, which involve a large number of parameters.

The standard EKF is known to suffer from linearization errors $([9,16-18])$. Several extensions of the standard EKF were proposed to remedy this situation as discussed next.

\subsection{Iterated EKF (iEKF)}

One way of reducing the linearization error in the estimation of the state $\hat{X}_{k+1}$ is to reformulate the Taylor series expansion of $g$ around the new estimate of $\hat{X}_{k+1}$, and recalculate the measurement 
update to get a better estimate of $\hat{X}_{k+1}$. The $l^{\text {th }}$ iteration of the measurement update equation for $\mathrm{iEKF}$ is given by ([7]):

$\left.\hat{X}_{k+1, l+1}=\hat{X}_{k+1}^{-}+K_{k+1, l} \mid z_{k+1}-g\left(\hat{X}_{k+1, l}, x_{k+1}, y_{k+1}\right)-G_{k+1, l}\left(\hat{X}_{k+1}^{-}-\hat{X}_{k+1, l}\right)\right\rfloor$

(4) Where

$G_{k+1, l}$ is the $g\left(\hat{X}_{k+1, l}\right)$ linearized about the current estimate $\hat{X}_{k+1, l}$

\subsection{Second-order EKF (SOEKF)}

As mentioned before, the main problem with the standard EKF is the linearization error which may result in the divergence of the filter. One simple alternative way to reduce this linearization error is to perform a second-order Taylor series expansion of the nonlinear models. The measurement update equations for the second-order EKF are given by [7]:

$$
\begin{aligned}
& \hat{X}_{k+1}=\hat{X}_{k+1}^{-}+K_{k+1}\left[z_{k+1}-g\left(\hat{X}_{k+1}^{-}, x_{k+1}, y_{k+1}\right)-\pi_{k+1}\right] \\
& \text { where, } \pi_{k+1}=\frac{1}{2} K_{k+1} \operatorname{Tr}\left[D_{k+1} P_{k+1}^{-}\right], \quad \text { and } D_{k+1}=\frac{\partial^{2} g}{\partial \hat{X}_{k+1}^{-2}}
\end{aligned}
$$

Note here that $\pi_{k}$ captures the contribution of the second-order term to the calculation of the state estimate $\hat{X}_{k+1} . D_{k+1}$ is the second-order of $g\left(\hat{X}_{k+1}^{-}, x_{k+1}, y_{k+1}\right)$ about the estimate $\hat{X}_{k+1}^{-}$.

\subsection{Standard Unscented KF (UKF)}

Unlike the EKF and its previously-discussed variants which rely on a deterministic truncated Taylor series approximation of the observation function (system model), the UKF relies on a form of statistical linearization which takes into account the probabilistic spread of the prior state estimate. Here a set of prior "sigma points" are first obtained through a deterministicallyweighted parameter vector and then nonlinearly-transformed by an unscented transform to generate a new set of posterior sigma points which are then used to estimate the posterior secondorder statistics (mean and covariance). A detailed description of the UKF can be found in the seminal work that first introduced this algorithm [14].

For our nonlinear transformation $z=g(X)$, we choose $(2 L+1)$ sigma points where $L$ is the dimension of $X$.

For the UKF measurement update equations, the sigma points and the nonlinear transformation are given by:

$$
\hat{X}_{k+1}^{(i)}=\hat{X}_{k+1}^{-}+\left(\sqrt{(L+\lambda) P_{k+1}^{-}}\right), \quad \hat{z}_{k+1}^{(i)}=g\left(\hat{X}_{k+1}^{(i)}, x_{k+1}, y_{k+1}\right)
$$

Where $\lambda=\alpha^{2}(L+\kappa)$, is a scaling parameter. The constant $\alpha$ determines the spread of the sigma points around $\bar{X}$, and the constant $\kappa$ is a secondary scaling parameter, $(\sqrt{(L+\lambda) P})_{i}$ is the $i^{\text {th }}$ column of the matrix square root obtained using the lower-triangular Cholesky factorization approach. 
Combining these $\hat{z}_{k+1}^{(i)}$ vectors to obtain the predicted measurement $\hat{z}_{k+1}$ yields:

$\hat{z}_{k+1}=\sum_{i=0}^{2 L} W^{(i)} \hat{z}_{k+1}^{(i)}$

Where the weights $W^{(i)}$ are given by:

$W^{(0)}=\frac{\lambda}{L+\lambda}, W^{(i)}=\frac{\lambda}{L+\lambda}+1-\alpha^{2}+\beta, \quad i=1,2, \cdots, 2 L$

\section{SIMULATION RESULTS}

As shown in Fig. 1, a 2D field $g(x, y)$ of size $m \times m=300 \times 300$ pixels is generated as the true field, which is to be reconstructed by sampling using a single mobile sensor. This true field is in fact the instantaneous state of the fire field we generated in our previous work using fire-spread models [5]. The acquisition of the initial estimate of the field is obtained by first dividing it into a uniform grid of size $n \times n=30 \times 30$, and then collecting an initial set of 100 samples, with each sample taken from the center of each cell of the grid. These samples are then used to train the neural network with $N=40$ neurons and a spread parameter of $\sigma=30$. This provides an initial estimate of the field with $1+4 \times 40=161$ parameters. A spot-measurement-based adaptive sampling scheme is then performed by the mobile sensor, considering points in a smaller grid of size $p \times p=5 \times 5$, in order to improve the field estimate.

It is important to point out at this juncture that the true field $g$ is estimated as $g_{\text {est }}$ which is obtained as follows: At every sample instant $k$, a measurement vector $z_{\mathrm{k}}$ is collected from which the field parameters are estimated. Then the field $\left(\mathrm{g}_{\text {est }}\right)$ is reconstructed from these estimated parameters. Two convergence criteria are used. First, the field estimation error, defined as the error between the original field $g$ and the estimated field $g_{\text {est }}$, is computed at every point $\left(x_{i}, y_{i}\right)$ of the field and then the 2-norm of this error is finally computed and used as a stopping criterion for validation purposes. In practice, although the original field is not known a priori, a denselysampled field can be used instead as an approximation of the field to be reconstructed.

The other criterion is the 2-norm of the parameter error covariance matrix $\left(\left\|P_{k+1}\right\|\right)$. This criterion is used for the selection of the next sample where the "expected" 2-norm of the error covariance of the parameter estimates, i.e. $\left(\left\|P_{k+1}\right\|\right)$, is calculated for various candidate locations, and the one which gives the least 2-norm $\left(\left\|P_{k+1}\right\|\right)$ is chosen as the "next sample". 
International Journal of Advanced Smart Sensor Network Systems (IJASSN), Vol 3, No.4,October 2013
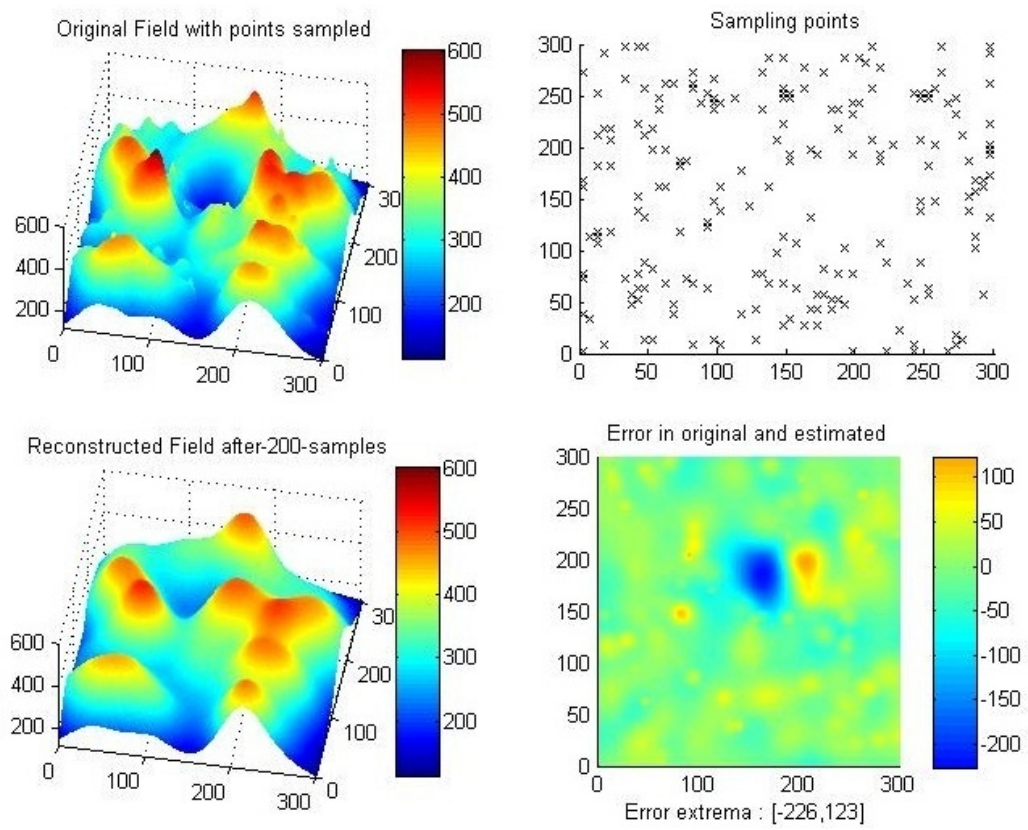

Figure 2. Simulation results for standard EKF showing original field, sampling points, reconstructed field, and the error in original and estimated field after 200 samples.

The estimate of the initial state vector formed with the field parameters in Eq. (1) is:

$$
\hat{X}_{0}=\left[\begin{array}{llllllllll}
\hat{b} & \hat{a}_{1} & \hat{\sigma}_{1} & \hat{x}_{01} & \hat{y}_{01} & \ldots & \hat{a}_{N} & \hat{\sigma}_{N} & \hat{x}_{0 N} & \hat{y}_{0 N}
\end{array}\right]_{0}^{T}
$$

Other assumptions are:

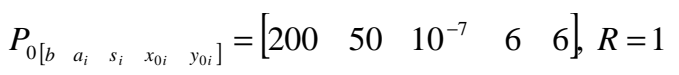

In Figure 2, the top left graph shows the true field whereas the top right one shows the sampling points when the standard EKF is used. In the bottom left graph, the reconstructed field is shown based on 200 samples only. The error between the true and estimated fields, after 200 samples, is shown in the bottom right graph. It is clear from this error graph that there is a huge error in one region where the error extremum is (-226). Figure 3 shows the results for the SOEKF after 200 samples for the same initial conditions as for the standard EKF above. The comparison between the 2 original and estimated field errors shown in Figures 2 and 3 respectively clearly shows that the large error extremum in the EKF case has been greatly reduced with the use of SOEKF. This is because the standard EKF is more prone to divergence than is the SOEKF. 
International Journal of Advanced Smart Sensor Network Systems (IJASSN), Vol 3, No.4,October 2013
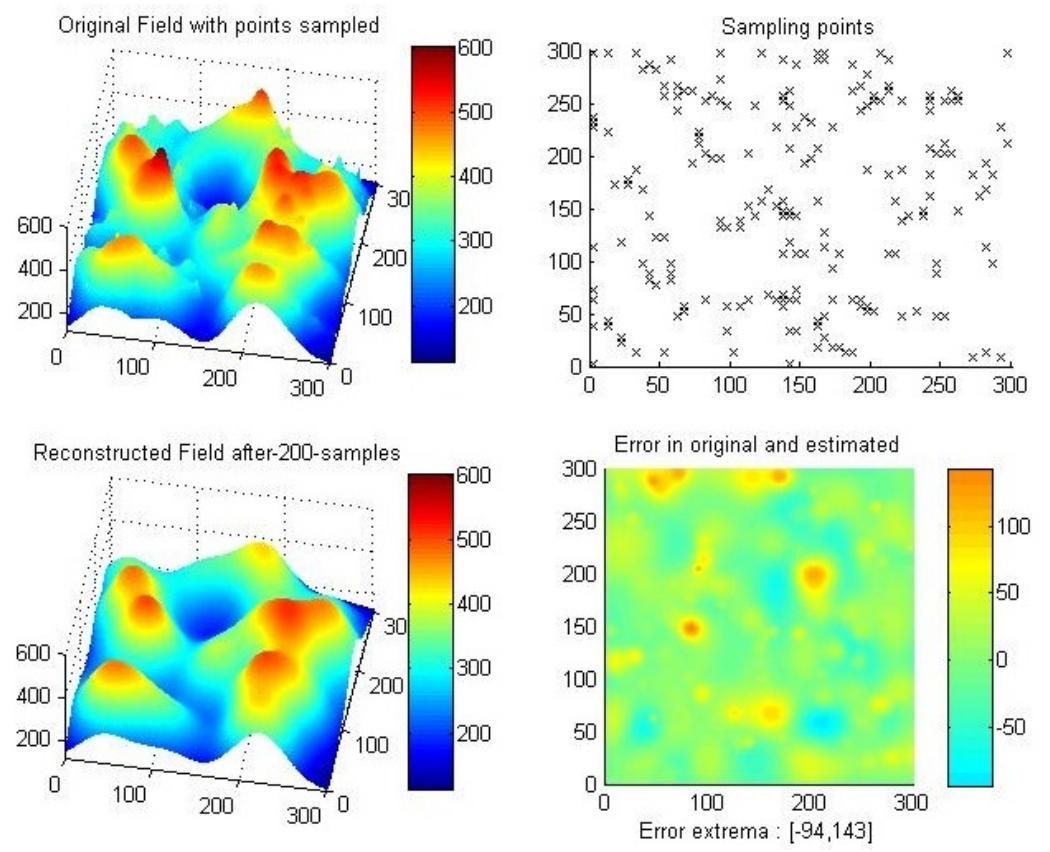

Figure 3. Simulation results for SOEKF showing original field, sampling points, reconstructed field, and the error in original and estimated field after 200 samples.

Figure 4 shows the performance of the standard EKF for various initial error covariance values for $\left(x_{0 i}, y_{0 i}\right)$ which are 4, 6 and 12. Figure 4 (a) and (b) show the 2-norm of the field error $\left(\left\|g-g_{\text {est } \_k+1}\right\|\right)$ and that of the error covariance of the parameter estimates i.e. $\left\|P_{k+1}\right\|$, respectively. It can be seen that a moderate increase in $P$ from 4 to 6 can lead to a noticeable increase in the norm over the sample range of (50-250). However, a large increase in P from 4 to 12 leads to a lesser increase in the norm value than that caused by the increase of $P$ from 4 to 6 , and is limited only to a narrower sample range of about (125-170). All three norms seem to eventually converge to the same steady-state value after 400 samples. It is important to note here that for some initial error covariance values (such as $P=10$ ), the filter diverges completely.
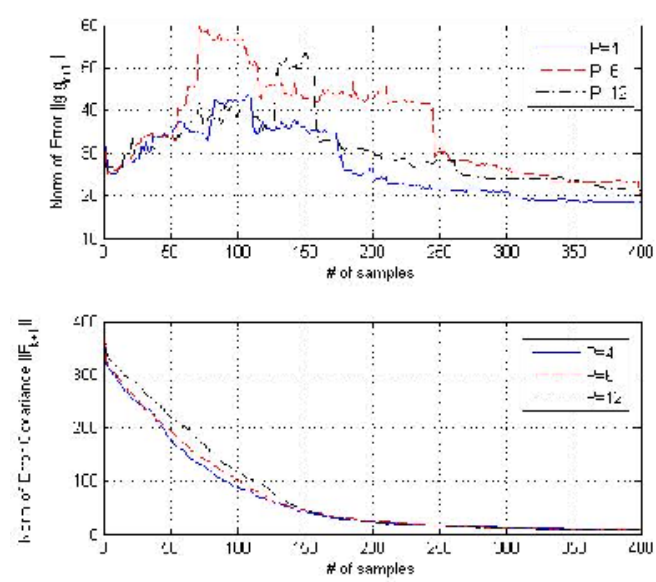
Figure 4. Simulation results showing the two errors when the standard EKF is used with 3 different initial error covariances for $\left(x_{0 i}, y_{0 i}\right)$
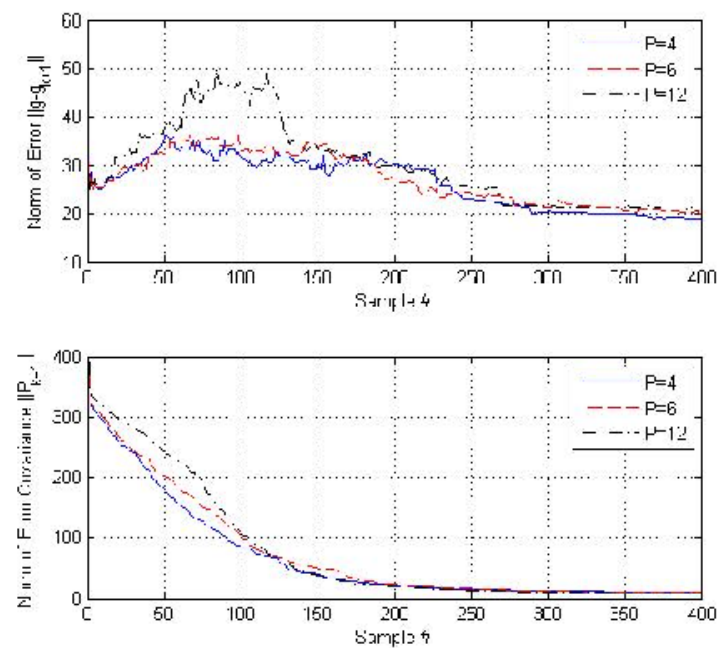

Figure 5. Simulation results showing the two errors when the Iterated EKF (with $n=5$ ) is used with 3 different initial error covariances for $\left(x_{0 i}, y_{0 i}\right)$

To mitigate the EKF's sensitivity to the initial values of the error covariance $P$, other filters are used as discussed next. Figures 5, 6 and 7 respectively show the results for iEKF $(n=5)$, SOEKF, and the standard UKF. The iEKF tries to avoid this divergence through a multiple application of the standard EKF equation as given in equation 5. But since the filter is of the first-order type, any resulting improvement is not very significant and the filter's divergence for $P=12$ could not be obviated. The divergence could also result from the accumulation of an unduly large initial linearization error.

Figure 6 shows the results achieved when the SOEKF filter is used. Note here that changing the initial error covariance does not result in any significant increase in the error norm. This is a clear indication that the SOEKF (eq. 5) does mitigate the problem of the EKF's sensitivity to the initial values of $\mathrm{P}$. It seems that the addition of the second-order term not only adds more accuracy to the state estimation process but also provides the "update" equation (eq. 5) with some "inertia" which then "slows down" the increase in the error norm. 
International Journal of Advanced Smart Sensor Network Systems (IJASSN), Vol 3, No.4,October 2013
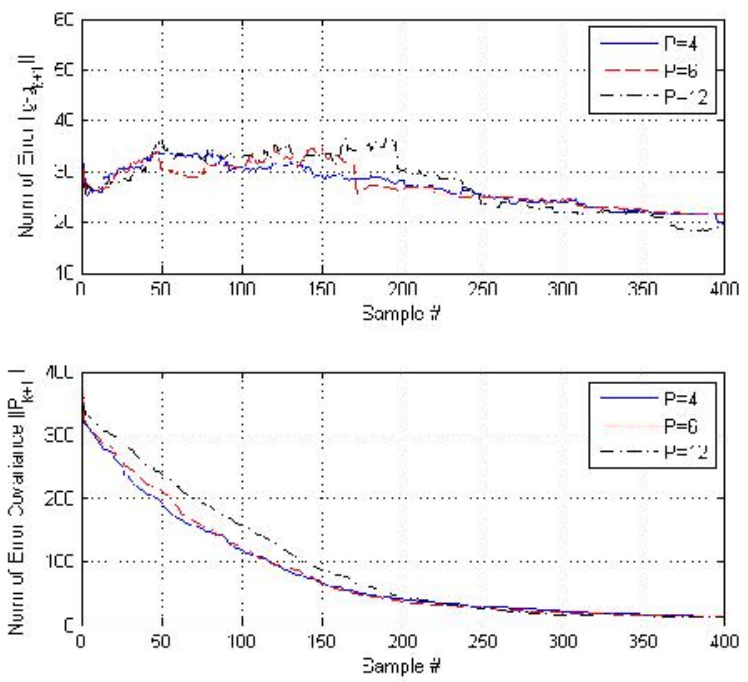

Figure 6. Simulation results showing the two errors when SOEKF is used with 3 different initial error covariances for $\left(x_{0 i}, y_{0 i}\right)$
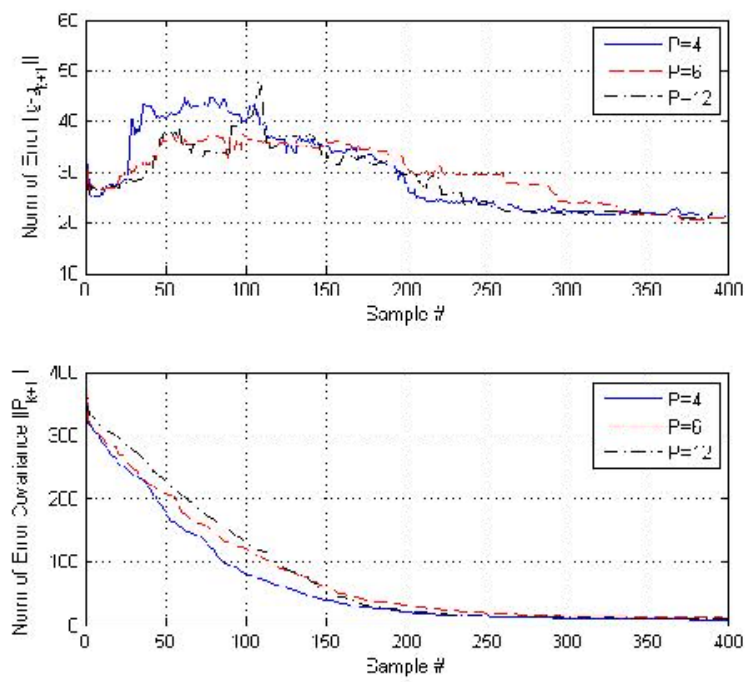

Figure 7. Simulation results showing the two errors when standard UKF is used with 3 different initial error covariances for $\left(x_{0 i}, y_{0 i}\right)$

Figure 7 shows the results for the UKF. In our simulations, we set $\alpha=10^{-4}$ and $\kappa=3-L$. Furthermore, another additive parameter $\beta$ is set to 2 in the aforementioned weighting of the sigma vectors in Eq. (8). UKF is less prone to divergence than the standard EKF because the former algorithm reduces the linearization errors involved in the latter one, by using its updated sets of sigma points to better capture the field state statistics through time.

In conclusion, the SOEKF has been shown to have the best performance for all initial values of the error covariance used here. 
Figure 8 shows a comparison between the performances of the standard EKF, iEKF ( $n=3$ and $n=5)$, SOEKF, and UKF for an initial error covariance value of 6 . The error covariance for the best-performing SOEKF decreases with the slowest pace, whereas that of the poorest-performing standard EKF decreases very fast. This shows that, for this value of $P=6$, the performance ranks from best to worst in the following order: SOEKF, iEKF ( $n=5), \operatorname{UKF}, \operatorname{iEKF}(n=3)$, standard EKF. The key aspects of the performance of these filters are summarized below in Table 1, where the metric used was the 2-norm of the field estimation error $\left(\left\|g-g_{\text {est }-k+1}\right\|\right)$.
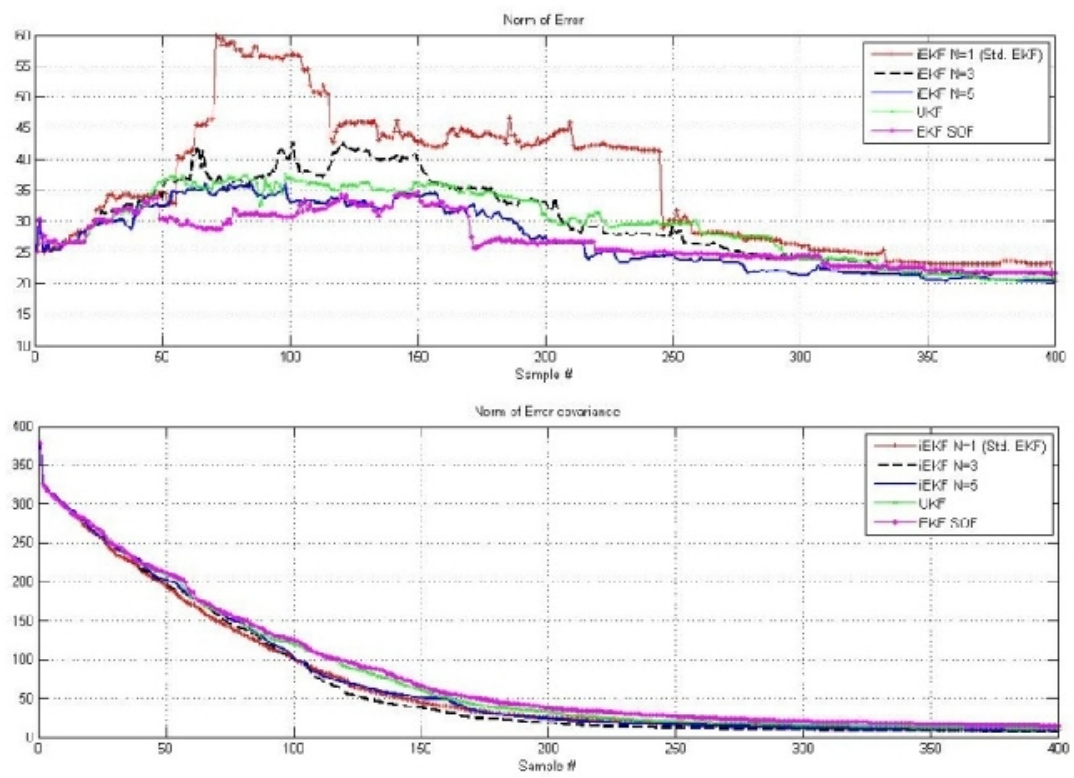

Figure 8. Simulation results showing the two errors for the Standard EKF, Iterated EKF (for $n=3$ and $n=5$ ), SOEKF, and UKF for 400 samples for an initial error covariance value of 6 for $\left(x_{0 i}, y_{0 i}\right)$.

To assess the impact of a poor initial field estimate, on the accuracy of the final field estimation, we considered a grid size of $40 \times 40$ which uses only a lower number of 49 samples to train the NN. As shown in figure 9, we have compared the performance of the EKF and SOEKF for the initial error covariance values of $P=6$ and $P=18$.

It can be seen from figure 9 that when an initial error covariance value of $P=18$ is assumed (thus putting less confidence in the initial field estimate), then the final field estimate obtained with both the EKF and SOEKF is better than the one obtained when the error covariance value of $P=6$ is assumed, which means that more confidence has been placed on the initial field estimate than is justified. For poor initial field estimates, a higher initial error covariance $P$ should be assumed for both the EKF and SOEKF, as it correctly reflects the higher uncertainty of the initial estimates. Moreover, for a poor initial field estimate, using a lower initial error covariance value of $P$ than is needed makes the error start to reduce quickly in the transient phase but, due to the low error covariance value used, ceases to reduce any further in the steady-state phase. This therefore shows that both the EKF and SOEKF are sensitive to the initial field estimate, with the SOEKF being less sensitive than the EKF.

It is worth pointing out here that this sensitivity issue is further emphasized in the previous case (figure 4) where the lower error covariance value of $P=4$ gave better results than for $P=6$ and $P=12$ for the EKF, because this lower value $(P=4)$ better reflects the uncertainty in the initial field 
estimate used than the other two values. As for the SOEKF and as shown in figure 6 , the value of the initial error covariance does not seem to have a significant effect on the final field estimate.
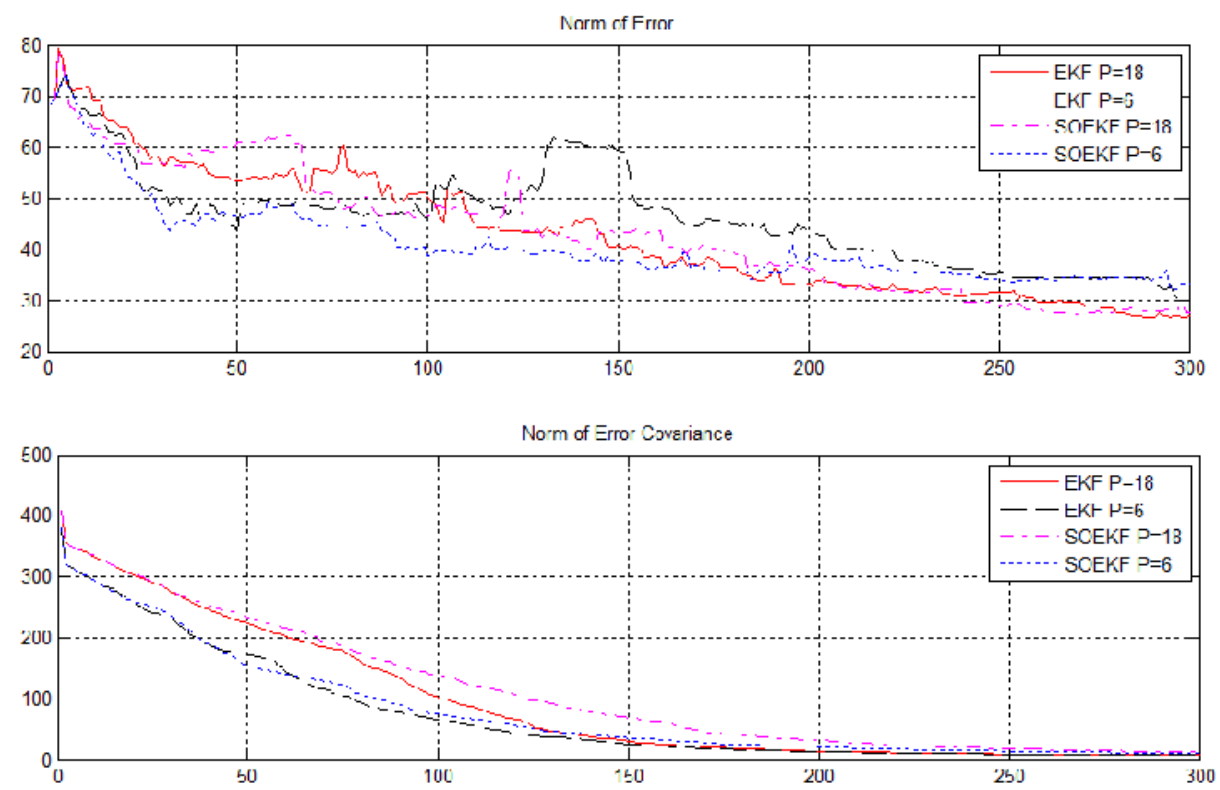

Figure 9. Simulation results showing the comparison of performance of EKF and SOEKF for two initial error covariance values, with poor initial field estimate

Table I shows the comparison of the performance of different filters in terms of sensitivity, accuracy and computational cost when the initial error covariance $P$ is varied by varying the location parameters $\left(\mathrm{x}_{0}, y_{0}\right)$ while keeping the other parameters as well as the initial field estimate values unchanged.

Table I: Performance of different filters considering sensitivity, accuracy and computational cost

\begin{tabular}{ll}
\hline $\begin{array}{l}\text { Sensitivity to initial P (Low to High) } \\
\text { [From figures 4-7] }\end{array}$ & SOEKF, iEKF (n=5), UKF, iEKF (n=3), EKF \\
\hline $\begin{array}{l}\text { Accuracy: (Error Low to High) } \\
\text { [From figure 8] }\end{array}$ & iEKF (n=5), SOEKF, iEKF (n=3), UKF, EKF \\
\hline $\begin{array}{l}\text { Computational Cost (Low to High) } \\
\text { [From eq. 4-7] }\end{array}$ & EKF, iEKF (n=3), iEKF (n=5), SOEKF, UKF \\
\hline
\end{tabular}

* Difference in accuracy of final results among SOEKF, iEKF $(n=3)$ and UKF is not very significant

\section{CONCLUSIONS AND FUTURE WORK}

In this paper, the interesting practical problem of estimating the spatial distribution of a field using traditional nonlinear filters (iEKF, SOEKF, UKF) supplemented with an adaptive sampling scheme performed by a single mobile sensor was addressed. Four traditional nonlinear filters, namely the standard EKF, iEKF, SOEKF and the standard UKF, were all studied and thoroughly tested on a novel application involving a large number of parameters. Specifically, this application consists of estimating a field distribution which, when parameterized, is represented 
with different initial error covariance values and different initial field estimates. It was shown that, from an accuracy point of view and with $P=6$, the four nonlinear filters can be ranked, from best to worst, as follows: SOEKF, $\operatorname{iEKF}(n=5), \operatorname{iEKF}(n=3), \mathrm{UKF}$ and EKF. It was shown that the SOEKF hardly exhibited any sensitivity to the choice of the initial value of the error covariance thanks to the "inertia" provided by the added second-order term in the state update equation. From a computational point of view, the UKF is more computationally intensive than all of the EKF, iEKF and SOEKF. The filter that seems to offer the best trade-off between estimation accuracy, sensitivity and computational load is either the iEKF $(n=5)$ or SOEKF, based on the initial values of the error covariance used here. The final choice of the estimation filter to use will depend on which of the issue (accuracy or sensitivity) is more critical to the application at hand. Although our comparison, in terms of the sensitivity to the initial field estimate, was limited to only the EKF and SOEKF, it showed however that in this regard, the SOEKF was less sensitive than EKF. Clearly, the performance of the UKF hinges upon 3 key features of the sigma points used: their number, the weights assigned to each point and the location of these points. By a judicious choice of some (or all) of these 3 important features, the well-known heavy computational load (due to large numbers of sigma points) associated with the standard UKF can be reduced. This challenge and the comparison of the sensitivity to the initial field estimate, of all of the 4 nonlinear filters used here, are the main focus of our future work. Finally, an interesting extension of this work would entail involving both the whole dynamics and noise characteristics (distribution and stationarity issues) of the true field to be reconstructed, rather than a single snapshot of it. The sensitivity analysis, limited here to the SOEKF and EKF only, would then be automatically subsumed in this extension work since the performance of the 4 filters would be evaluated as the true field evolves in time.

\section{ACKNOWLEDGEMENTS}

The authors would like to acknowledge the support provided by the Deanship of Scientific Research (DSR) at King Fahd University of Petroleum \& Minerals (KFUPM) for funding this work through project no. SB101017.

\section{REFERENCES}

[1] G. Anastasi, M. Conti, M. Di Francesco, A. Passarella, "Energy Conservation in Wireless Sensor Networks: a Survey”, Ad Hoc Networks, Vol. 7, N. 3, pp. 537-568, May 2009. Elsevier.

[2] V.N. Christopoulos and S. Roumeliotis, "Adaptive Sensing for Instantaneous Gas Release Parameter Estimation," in IEEE International Conference on Robotics and Automation, 2005, pp. 4450-4456.

[3] C.J. Cannell and D.J. Stilwell, "A Comparison of Two Approaches for Adaptive Sampling of Environmental Processes Using Autonomous Underwater Vehicles," in Proceedings of MTS/IEEE OCEANS, 2005, pp. 1514-1521.

[4] R. Graham, J. Cortes, "Cooperative adaptive sampling via approximate entropy maximization" Decision and Control, 2009 held jointly with the 2009 28th Chinese Control Conference. CDC/CCC 2009. Proceedings of the 48th IEEE Conference, pp.7055-7060, 15-18 Dec. 2009.

[5] M. F. Mysorewala, D. O. Popa, "Multi-scale Adaptive Sampling with Mobile Agents for Mapping of Forest Fires", in the Journal of Intelligent and Robotic Systems, vol. 54, no. 4, April 2009, pages 535565.

[6] D. O. Popa; M. F. Mysorewala; F. L. Lewis, "EKF-based Adaptive Sampling with Mobile Robotic Sensor Nodes", International Conference on Intelligent Robots and Systems, 2006 IEEE/RSJ, pp. 2451-2456, Oct. 2006.

[7] Dan Simon, "Optimal State Estimation: Kalman, H-Infinity, and Nonlinear Approaches", WileyInterscience, 2006.

[8] T. Lefebvre et. Al.: "Nonlinear Kalman Filtering”, STAR 19, pp. 51-76, 2005, Springer-Verlag Berlin Heidelberg, 2005. 
International Journal of Advanced Smart Sensor Network Systems (IJASSN), Vol 3, No.4,October 2013

[9] L. Perea, J. How, L. Breger, P. Elosegui, "Nonlinearity in sensor fusion: Divergence issues in EKF, modified truncated SOF, and UKF", Proc. AIAA Guid., Navig., Control Conf., pp. 2007.

[10] Ningzhou Cui, Lang Hong, Jeffery R. Layne, “A comparison of nonlinear filtering approaches with an application to ground target tracking", Signal Processing, Volume 85, Issue 8, August 2005, Pages 1469-1492.

[11] Monica F. Bugallo, Shanshan Xu, Petar M. Djuric, "Performance comparison of EKF and particle filtering methods for maneuvering targets", Digital Signal Processing, Volume 17, Issue 4, July 2007, Pages 774-786.1

[12] Y. Bar-Shalom, X.R. Li, Estimation and Tracking: Principles, Techniques, and Software, Artech House, Norwood, MA, 1993.

[13] M. F. Mysorewala, L. Cheded, M. S. Baig, D. O. Popa , "A distributed multi-robot adaptive sampling scheme for complex field estimation", in 11th International Conference on Control, Automation, Robotics \& Vision (ICARCV), 2010, pp.2466-2471, 7-10 Dec. 2010.

[14] E. A. Wan, R. Van Der Merwe,"The unscented Kalman filter for nonlinear estimation", Adaptive Systems for Signal Processing, Communications, and Control Symposium 2000. AS-SPCC. The IEEE 2000 , vol., no., pp.153-158, 2000.

[15] S. K. Thompson, "Sampling", Wiley-Interscience, 2002.

[16] T. Lefebvre, H. Bruyninckx, and J. D. Schutter, "Kalman filters for nonlinear systems: A comparison of performance," Internal Report 01R033 ME Dept. Katholieke Universiteit Leuven, Belgium, 2001.

[17] T. Lefebvre, H. Bruyninckx, J.D. Schutter, "4 Kalman Filters for Nonlinear Systems," in Nonlinear Kalman Filtering for Force-Controlled Robot Tasks, vol. 19, pp. 51-76, Springer, 2005.

[18] Y. Bar-Shalom, X. Li, and T. Kirubarajan, Estimation with Applications to Tracking and Navigation: Theory, Algorithms and Software. John Wiley \& Sons, 2001. 